\title{
Impact of trait anxiety on psychological well-being in men with prostate cancer
}

\author{
Rikiya Taoka', Hisato Matsunaga², Tatsuhiko Kubo ${ }^{3}$, Toru Suzuki', Shingo Yamamoto ${ }^{1}$ \\ ${ }^{1}$ Department of Urology, Hyogo College of Medicine, Japan; ${ }^{2}$ Department of Neuropsychiatry, Hyogo \\ College of Medicine, Japan; ${ }^{3}$ Department of Public Health, University of Occupational and Environmental \\ Health, Japan
}

\section{ABSTRACT}

Objective: The aim of the present study was to determine state anxiety following radical treatment for localized prostate cancer (PCa), and the impact of trait anxiety on psychological well-being in affected patients.

Material and Methods: The present study was a cross-sectional survey of 70 men with localized PCa performed between February 2012 and July 2012. Of those, 21, 25, and 24 patients were treated by radical retropubic prostatectomy (RRP), permanent prostate brachytherapy (PPB), and external beam radiotherapy (EBRT), respectively. State anxiety, trait anxiety, and general health were assessed using the State-Trait Anxiety Inventory and 8 Items Short Form Health Survey (SF-8).

Results: The rate of very high and high state anxiety in patients who received RRP was 47.6\%, while that in patients who received PPB and EBRT was 40.0\% and 37.5\%, respectively. In contrast, the rate of very high and high trait anxiety in the RRP group was much lower (23.7\%). Trait anxiety showed a high correlation with state anxiety and the mental health component summary of SF-8 (correlation coefficient=0.715, -0.504). Conclusions: Trait anxiety was associated with the degree of state anxiety regarding treatments for PCa, followed by change in state anxiety, which might have effects on psychological well-being. Information regarding state anxiety as a consequence of treatments and trait anxiety measurement tool are important considerations for treatment decision-making in newly diagnosed PCa patients.

\section{ARTICLE INFO}

\section{Key words:}

Prostatic Neoplasms; Quality of Life; Anxiety

Int Braz J Urol. 2014; 40: 620-6

Submitted for publication:

February 09, 2014

Accepted after revision:

April 04, 2014

\section{INTRODUCTION}

The incidence rate of localized prostate cancer ( $\mathrm{PCa}$ ) has been increasing (1), mainly because of the wide availability of prostate specific antigen (PSA) screening (2). The majority of men with localized PCa primarily undergo some form of radical treatment, such as radical retropubic prostatectomy (RRP), external beam radiotherapy (EBRT), or permanent prostate brachytherapy (PPB) (3). However, since RRP and radiotherapy achieve similar oncological outcomes in most patients (4), they are often troubled by treatment decision-making for newly diagnosed localized PCa.

Each of those radical treatments is associated with a distinct profile of longitudinal health-related quality of life (HRQOL) (5). Thus, HRQOL measures are an important consideration for deciding treatment. Recently, a number of studies have reported HRQOL in cases of localized PCa with the aim of providing information concerning the potential consequences of treatments (6-8). 
However, despite increased focus on disease-specific HRQOL, less attention has been given to the psychological well-being of affected patients.

Anxiety is defined as a state of apprehension and fear arising from anticipation of a threatening event, and it often impairs physical and psychological functions (9). A recent meta-analysis (10) revealed that anxiety was most likely to be a problem in cancer survivors. The present study investigated anxiety using State-Trait Anxiety Inventory (STAI) (11), a widely used questionnaires to measure anxiety, in patients being treated for localized PCa. In particular, this tool can measure both state anxiety (how anxious a person is feeling at a particular moment) and trait anxiety (how dispositionally anxious a person is across time and situations). The aim of the present study was to determine state anxiety following each radical treatment for localized PCa, and the impact of trait anxiety on psychological well-being and treatment decision-making in affected patients.

\section{MATERIALS AND METHODS}

The present study was performed as a cross-sectional survey of 70 men with localized PCa being treated in our outpatient clinic between February and July 2012. Of those, 21, 25, and 24 were treated by RRP, PPB, and EBRT, respectively. Exclusion criteria were past history of any neuropsychiatric disorder or any psychoactive drug use, and progressive disease. Anxiety and general health were assessed using self-report questionnaires. Approval for data collection in this comprehensive assessment was provided by the Institutional Review Board of our institution (No. 1163) and written informed consent was obtained from each patient.

The questionnaires consisted of two different instruments, with the Japanese version of each confirmed for reliability and validity $(12,13)$. First, anxiety was measured using the STAI (12), which consists of two separate sub-scales (state and trait anxiety) containing 20 items each. Each item is scored from 1 to 4 , with the total score ranging from 20 to 80 for each scale and high scores indicating increased anxiety. In addition, results of the STAI can be divided into 5 degrees of trait and state anxiety, including very low, low, normal, high, and very high. Second, general health was assessed using the 8 Items Short Form Health Survey (SF-8) (13). This questionnaire is comprised of 8 sub-scales, each of which measures a different health dimension; physical function, role-physical, bodily pain, general health, vitality, social function, mental health, and role-emotional. The SF-8 generates two summary scores, the physical health component summary (PCS) and mental health component summary (MCS). In the present study, the score for each of the 8 subscales, and PCS and MCS were determined using the Norm-Based Scoring method, which is based on a large-scale population study conducted in Japan (13).

Comparisons of clinical characteristics and each SF-8 score among the 3 groups were made using a Kruskal-Wallis or Chi-square test. Correlations of trait anxiety with state anxiety, PCS, and MCS were examined by Spearman's rank correlation. All statistical analyses were performed using SPSS version 12 for Windows (SPSS Inc, Chicago, IL). P-values less than 0.05 were considered to indicate statistical significance.

\section{RESULTS}

Table- 1 shows the clinical characteristics of the 70 patients. There were no significant differences in regard to follow-up term at time of the survey among the 3 groups ( $p=0.233)$. In contrast, age at treatment in the RRP group was younger than that in the PPB and EBRT groups $(p=0.005)$. The mean value for PSA at diagnosis in the PPB group was lower than in the other groups ( $<<0.001)$. In addition, clinical diagnosis and Gleason sum in the PPB group were the lowest among the groups $(p=0.007,<0.001)$. In the present study, the definition of biochemical recurrence differed between prostatectomy cases (two consecutive PSA values of $0.2 \mathrm{ng} / \mathrm{mL}$ or greater (14)) and radiation cases (elevation of $2 \mathrm{ng} / \mathrm{mL}$ above post-treatment PSA-nadir (15)). Based on those definitions, 6 patients were diagnosed with biochemical recurrence, and no local recurrence or distant metastasis was confirmed. Table- 2 presents scores of the 8 subscales and 2 component summaries of the SF- 8 for outpatients who received radical treatment for lo- 
Table 1 - Clinical characteristics of study population.

\begin{tabular}{|c|c|c|c|c|c|c|c|c|}
\hline & & \multicolumn{2}{|c|}{$\begin{array}{c}\text { RRP } \\
(n=21)\end{array}$} & \multicolumn{2}{|c|}{$\begin{array}{c}\text { PPB } \\
(n=25)\end{array}$} & \multicolumn{2}{|c|}{$\begin{array}{c}\text { EBRT } \\
(n=24)\end{array}$} & $p$-value \\
\hline Age at treatment (years) & & \multicolumn{2}{|c|}{$65.3 \pm 7.8$} & \multicolumn{2}{|c|}{$68.7 \pm 5.3$} & \multicolumn{2}{|c|}{$72.0 \pm 5.2$} & $p=0.005^{\dagger}$ \\
\hline Follow up at survey (month) & & \multicolumn{2}{|c|}{$12.5 \pm 18.1$} & \multicolumn{2}{|c|}{$15.6 \pm 13.1$} & \multicolumn{2}{|c|}{$14.8 \pm 13.4$} & $p=0.233^{\dagger}$ \\
\hline PSA at diagnosis ( $\mathrm{ng} / \mathrm{mL})$ & & \multicolumn{2}{|c|}{$13.2 \pm 7.3$} & \multicolumn{2}{|c|}{$6.7 \pm 2.6$} & \multicolumn{2}{|c|}{$21.0 \pm 33.5$} & $p<0.001^{\dagger}$ \\
\hline \multirow[t]{3}{*}{ Clinical diagnosis } & $\mathrm{T} 1 \mathrm{c}$ & 6 & (28.6) & 13 & $(52.0)$ & 8 & (33.3) & \\
\hline & T2 & 14 & $(66.7)$ & 12 & $(48.0)$ & 9 & (37.5) & $p=0.007^{\ddagger}$ \\
\hline & ТЗа & 1 & $(4.7)$ & 0 & (0) & 7 & $(29.2)$ & \\
\hline \multirow[t]{3}{*}{ Gleason sum } & $\leq 6$ & 6 & (28.6) & 13 & $(52.0)$ & 5 & $(20.8)$ & \\
\hline & 7 & 12 & $(57.1)$ & 12 & $(48.0)$ & 7 & $(29.2)$ & $p<0.001^{\ddagger}$ \\
\hline & $\geq 8$ & 3 & (14.3) & 0 & $(0)$ & 12 & $(50.0)$ & \\
\hline Recurrence & & 3 & $(14.3)$ & 0 & $(0)$ & 3 & (12.5) & \\
\hline
\end{tabular}

Data are presented as mean $\pm \mathrm{SD}$ or $\mathrm{N}(\%)$. $†$ Kruskal-Wallis test; $\ddagger$ Chi-square test.

Table 2 - Subscales and component summaries of SF-8 in 3 treatment groups.

\begin{tabular}{|c|c|c|c|c|c|c|c|c|c|c|}
\hline & RRP & & & PPB & & & EBRT & & & $p^{-v a l u e}$ \\
\hline General health & 51.7 & \pm & 6.1 & 51.3 & \pm & 8.5 & 48.9 & \pm & 8.4 & $p=0.493$ \\
\hline Physical functioning & 46.4 & \pm & 9.6 & 48.9 & \pm & 5.9 & 46.7 & \pm & 9.1 & $p=0.607$ \\
\hline Role physical & 47.2 & \pm & 8.2 & 49.5 & \pm & 8.9 & 46.9 & \pm & 9.9 & $p=0.315$ \\
\hline Bodily pain & 53.1 & \pm & 7.8 & 52.3 & \pm & 7.5 & 53.6 & \pm & 7.3 & $p=0.678$ \\
\hline Vitality & 52.9 & \pm & 6.2 & 52.0 & \pm & 6.5 & 50.9 & \pm & 7.9 & $p=0.876$ \\
\hline Social functioning & 47.8 & \pm & 9.8 & 50.2 & \pm & 6.9 & 49.0 & \pm & 7.6 & $p=0.741$ \\
\hline Mental health & 49.8 & \pm & 5.8 & 51.4 & \pm & 6.0 & 50.4 & \pm & 8.0 & $p=0.574$ \\
\hline Role emotional & 48.4 & \pm & 9.8 & 49.8 & \pm & 7.2 & 48.8 & \pm & 7.3 & $\mathrm{p}=0.858$ \\
\hline PCS & 48.3 & \pm & 7.7 & 49.0 & \pm & 6.5 & 47.4 & \pm & 8.2 & $p=0.934$ \\
\hline MCS & 49.1 & \pm & 6.7 & 50.3 & \pm & 6.1 & 49.5 & \pm & 7.6 & $p=0.909$ \\
\hline
\end{tabular}

Data are presented as mean $\pm S D$. † Kruskal-Wallis test. PCS; physical health component summary, MCS; mental health component summary.

calized PCa. There were no significant differences among the 3 groups for any of those scores.

Figure-1A shows state anxiety in our 70 patients who received radical treatments for localized PCa. It is noteworthy that $29(41.4 \%)$ had very high or high state anxiety. The rate of very high and high state anxiety in those who received RRP was $47.6 \%$, while that in patients who received PPB or EBRT was $40.0 \%$ and $37.5 \%$, respectively. Figure-1B shows trait anxiety in these patients. The rate for very 
Figure 1 - State anxiety and trait anxiety in patients who received radical treatments for localized PCa are shown in a and b, respectively. Twenty-nine of the 70 patients $(41.4 \%)$ had very high or high state anxiety. The degree of state anxiety in patients who received RRP was highest among the 3 groups, while that of trait anxiety was lowest.

A

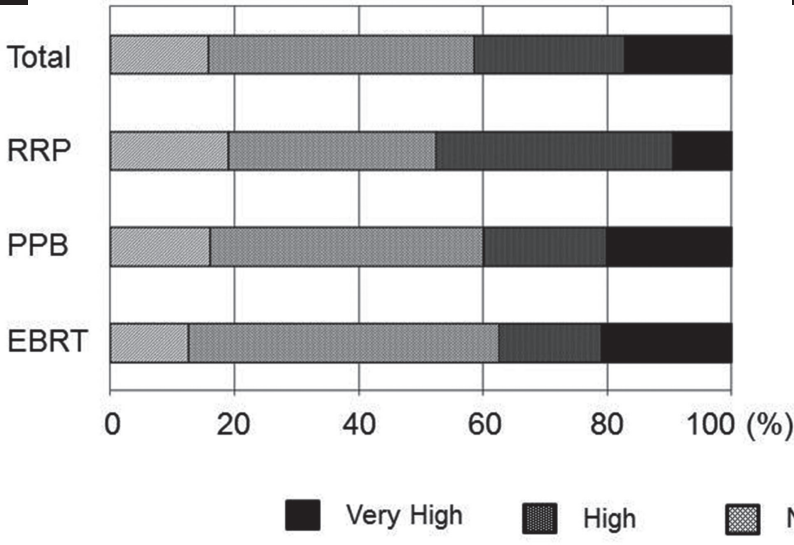

B

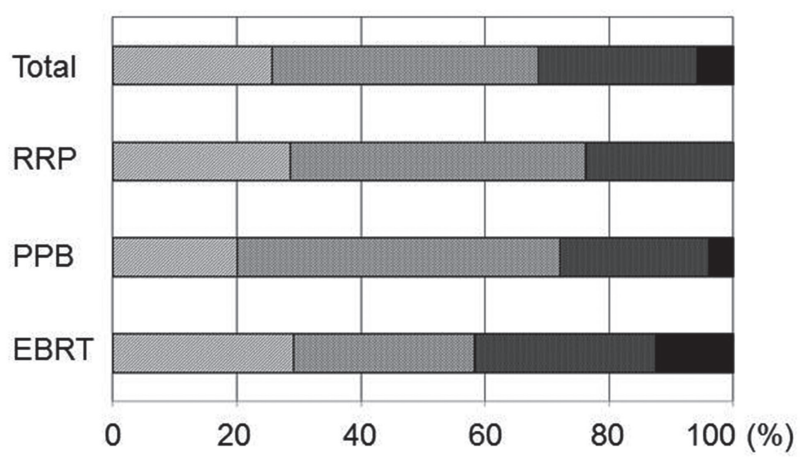

Normal $\quad \square$ Low $\square$ Very Low high and high trait anxiety in all patients was 31.4\%. Notably, only 5 (23.8\%) in the RRP group showed very high or high trait anxiety, while $10(41.7 \%)$ in the EBRT group had very high or high trait anxiety.

Figure 2A, B, and C show correlations of trait anxiety with state anxiety, PCS, and MCS, as determined by Spearman's rank correlation coefficient. The coefficient of determination was 0.715 for trait anxiety and state anxiety, -0.352 for trait anxiety and PCS, and -0.504 for trait anxiety and MCS. The remarkably high coefficient between trait anxiety and state anxiety indicated that trait anxiety was strongly correlated to state anxiety in our patients. In addition, the correlation of trait anxiety with MCS was stronger than with PCS.

\section{DISCUSSION}

Recently, a number of prospective studies have investigated erectile dysfunction, incontinence, bowel urgency, and other adverse effects following treatment with RRP, EBRT, and PPB $(6,7)$. In addition, the relationship between post-treatment problems and HRQOL was examined (8). However, despite increased focus on physical problems following those treatments, little attention has been given to related psychological distress. A large population based study (16) showed that the prevalence rate of psychological distress in cancer patients was 35.1\%. However, psychological distress often goes unrecognized (17) and can have negative implications for patients including reduced HRQOL (18). The present findings revealed that the rate of very high or high state anxiety was $41.4 \%$ in patients with localized PCa following radical treatments. A recent meta-analysis (10) reported that anxiety was most likely to be a problem in cancer survivors. Physicians should recognize the importance of screening for state anxiety in men with PCa.

A recent meta-analysis (19) showed that the prevalence of anxiety in patients with PCa were different across the different treatment stages. Interestingly, the prevalence of anxiety in patients who have completed treatment was higher than that in patients who were currently undertaking treatment. The increased anxiety following radical treatment for PCa could be related to a fear of recurrence (20). In particular, it was reported that PSA testing was associated with a significant amount of anxiety (20). Therefore, it is thought that the periodical screening for state anxiety following radical treatment is necessary to minimize the impact of anxiety on psychological well-being.

Psychological well-being in patients is associated with adverse effects following radical treatments for PCa. It was reported that the degree of anxiety in patients who received RRP was associa- 
Figure 2 - Correlations of trait anxiety with state anxiety, PCS, and MCS are shown (Spearman's rank correlation coefficient) (A, B, C). Trait anxiety was strongly correlated to state anxiety. In addition, the correlation of trait anxiety with MCS was stronger than that with PCS.

A

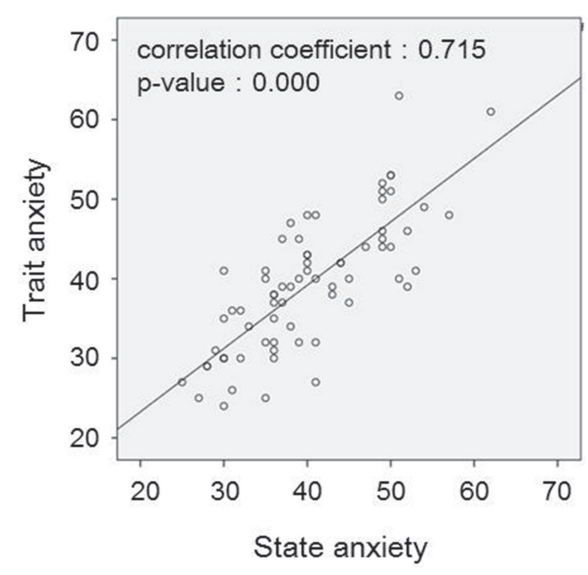

B

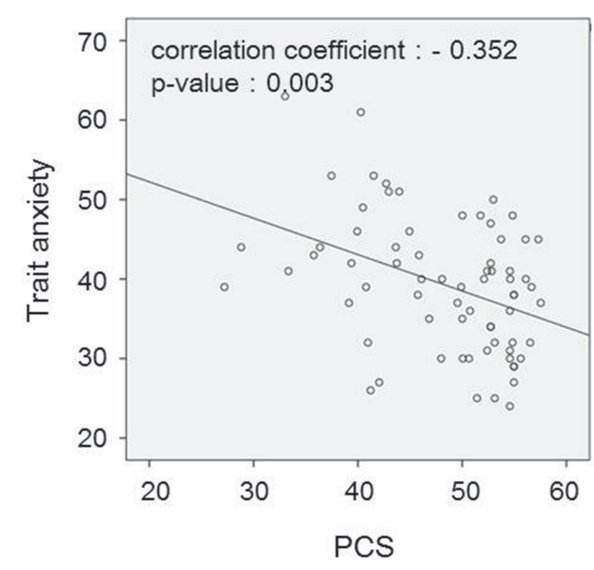

c

(c)

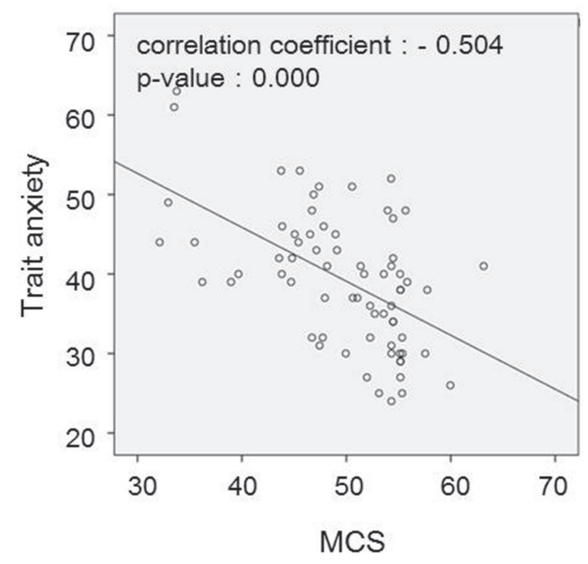

ted with sexual outcome (21). In addition, patients who were experiencing urinary and bowel symptoms tended to suffer from moderate to higher distress compared with patients reporting no or fewer such symptoms (22). Furthermore, the presence of comorbid psychiatric conditions was reported to be a risk factor related to poor HRQOL in patients after treatment (23). In the present study, we found a robust correlation between the elevation of trait anxiety and that of state anxiety. Among the 3 groups, trait anxiety was lowest and state anxiety was highest in patients who received RRP, suggesting that those with high trait anxiety should avoid RRP. Our findings indicate that trait anxiety measurement is an important tool for treatment decision-making for newly diagnosed PCa patients.

The increase in life expectancy of patients with localized PCa has highlighted HRQOL after treatment as a key issue. Our results revealed that trait anxiety is associated with the degree of state anxiety in regard to treatments for PCa, followed by change in state anxiety, which might have effects on psychological well-being. Therefore, the present study shows the potential of assessment of trait anxiety predicts post-treatment psychological well-being. However, our analysis has some limitations. First, there is no significant difference in comparison of state anxiety and trait anxiety among 3 groups. Second, the present study had a relatively small sample size that might not have statistical power sufficient to show correlations of trait anxiety with state anxiety, PCS, and MCS. Third, due to the cross-sectional nature of the present study, there were no pre-treatment STAI data available. Therefore, we were unable to investigate the change in state anxiety in regard to radical treatments for localized PCa. On the other hand, trait anxiety is a personal characteristic defined as feelings of stress, worry, and discomfort (11). As a result, such pre-treatment data may not be necessary, because there are likely minimal changes. Nevertheless, a large scale longitudinal study that includes pre-treatment data may be useful for gathering additional information about anxiety regarding radical treatments for localized PCa. Information in regard to state anxiety related to the outcome of treatments and trait anxiety measurements are anticipated to become important 
considerations for treatment decision-making for newly diagnosed PCa patients.

\section{CONCLUSIONS}

In the present study we utilized the STAI, a widely used questionnaire to assess the types and degree of anxiety in patients undergoing radical treatments for localized PCa. Our results indicate that trait anxiety is associated with the degree of state anxiety in regard to treatments for PCa, followed by change in state anxiety, which might have effects on psychological well-being. The present study shows the potential of assessment of trait anxiety to predict post-treatment psychological well-being.

\section{ABBREVIATIONS}

$\mathrm{PCa}=$ Prostate cancer

PSA $=$ Prostate specific antigen

$\mathrm{RRP}=$ Radical retropubic prostatectomy

EBRT $=$ External beam radiotherapy

PPB = Permanent prostate brachytherapy

HRQOL = Health-related quality of life

STAI = State-Trait Anxiety Inventory

PCS = Physical health component summary

MCS $=$ Mental health component summary

\section{FINANCIAL DISCLOSURE}

This work was supported by a Grant-in-Aid for Researchers, Hyogo College of Medicine, 2011.

\section{CONFLICT OF INTEREST}

None declared.

\section{REFERENCES}

1. Cooperberg MR, Broering JM, Kantoff PW, Carroll PR. Contemporary trends in low risk prostate cancer: risk assessment and treatment. J Urol. 2007;178(3 Pt 2):S14-9.

2. Aus G, Bergdahl S, Lodding P, Lilja H, Hugosson J. Prostate cancer screening decreases the absolute risk of being diagnosed with advanced prostate cancer--results from a prospective, population-based randomized controlled trial. Eur Urol. 2007;51:659-64.
3. Cooperberg MR, Broering JM, Carroll PR. Time trends and local variation in primary treatment of localized prostate cancer. J Clin Oncol. 2010;28:1117-23.

4. Zietman AL, Chung CS, Coen JJ, Shipley WU. 10-year outcome for men with localized prostate cancer treated with external radiation therapy: results of a cohort study. J Urol. 2004;171:210-4.

5. Sanda MG, Dunn RL, Michalski J, Sandler HM, Northouse L, Hembroff L, et al. Quality of life and satisfaction with outcome among prostate-cancer survivors. N Engl J Med. 2008;358:1250-61.

6. Talcott JA, Manola J, Clark JA, Kaplan I, Beard CJ, Mitchell SP, et al. Time course and predictors of symptoms after primary prostate cancer therapy. J Clin Oncol. 2003;21:3979-86.

7. Pardo Y, Guedea F, Aguiló F, Fernández P, Macías V, Mariño $A$, et al. Quality-of-life impact of primary treatments for localized prostate cancer in patients without hormonal treatment. J Clin Oncol. 2010;28:4687-96. Erratum in: J Clin Oncol. 2011;29:779.

8. Kyrdalen AE, Dahl AA, Hernes E, Småstuen MC, Fosså SD. A national study of adverse effects and global quality of life among candidates for curative treatment for prostate cancer. BJU Int. 2013;111:221-32.

9. Khan H, Kalia S, Itrat A, Khan A, Kamal M, Khan MA, et al. Prevalence and demographics of anxiety disorders: a snapshot from a community health centre in Pakistan. Ann Gen Psychiatry. 2007;6:30

10. Mitchell AJ, Ferguson DW, Gill J, Paul J, Symonds P. Depression and anxiety in long-term cancer survivors compared with spouses and healthy controls: a systematic review and meta-analysis. Lancet Oncol. 2013;14:721-32.

11. Spielberger CD, Gorsuch RI, Lushene RE: STAI Manual for the State-Trait Anxiety Inventory. Palo Alto: Consulting Psychologist Press. 1970;23-49.

12. Nakazato K, Mizuguchi T: Development and validation of the Japanese version of the State-Trait Anxieth Inventory. Shinshin-Igaku. 1982; 22:107-112.

13. Fukuhara S, Suzukamo Y: Manual of the SF-8 Japanese Version: Institute for Health Outcome and Process Evaluation Research, Kyoto, 2004.

14. Moul JW. Prostate specific antigen only progression of prostate cancer. J Urol. 2000;163:1632-42.

15. Roach M 3rd, Hanks G, Thames H Jr, Schellhammer P, Shipley WU, Sokol GH, et al. Defining biochemical failure following radiotherapy with or without hormonal therapy in men with clinically localized prostate cancer: recommendations of the RTOG-ASTRO Phoenix Consensus Conference. Int J Radiat Oncol Biol Phys. 2006;65:965-74.

16. Zabora J, BrintzenhofeSzoc K, Curbow B, Hooker C, Piantadosi S. The prevalence of psychological distress by cancer site. Psychooncology. 2001;10:19-28. 
17. Fallowfield L, Ratcliffe D, Jenkins V, Saul J. Psychiatric morbidity and its recognition by doctors in patients with cancer. Br J Cancer. 2001;84:1011-5.

18. Shim EJ, Shin YW, Jeon HJ, Hahm BJ. Distress and its correlates in Korean cancer patients: pilot use of the distress thermometer and the problem list. Psychooncology. 200817:548-55.

19. Watts S, Leydon G, Birch B, Prescott P, Lai L, Eardley S, et al. Depression and anxiety in prostate cancer: a systematic review and meta-analysis of prevalence rates. BMJ Open. 2014;4:e003901.

20. De Sousa A, Sonavane S, Mehta J. Psychological aspects of prostate cancer: a clinical review. Prostate Cancer Prostatic Dis. 2012;15:120-7.

21. Punnen S, Cowan JE, Dunn LB, Shumay DM, Carroll PR, Cooperberg MR. A longitudinal study of anxiety, depression and distress as predictors of sexual and urinary quality of life in men with prostate cancer. BJU Int. 2013;112:E67-75.
22. Namiki S, Saito S, Tochigi T, Numata I, Ioritani N, Arai Y. Psychological distress in Japanese men with localized prostate cancer. Int J Urol. 2007;14:924-9.

23. Schag CA, Ganz PA, Wing DS, Sim MS, Lee JJ. Quality of life in adult survivors of lung, colon and prostate cancer. Qual Life Res. 1994;3:127-41.

\section{Correspondence address:}

Rikiya Taoka, MD

Department of Urology, Hyogo College of Medicine, 1-1, Mukogawa-cho, Nishinomiya, Hyogo, 663-8501, Japan

FAX: +81 798 456-368

E-mail: rtaoka@mdanderson.org 